\title{
Peen treatment on a titanium implant: effect of roughness, osteoblast cell functions, and bonding with bone cement
}

This article was published in the following Dove Press journal:

International Journal of Nanomedicine

4 February 2016

Number of times this article has been viewed

\section{Morshed Khandaker ${ }^{1,4}$ \\ Shahram Riahinezhad' \\ Fariha Sultana' \\ Melville B Vaughan ${ }^{2,4}$ \\ Joshua Knight ${ }^{2}$ \\ Tracy L Morris ${ }^{3,4}$}

'Department of Engineering \& Physics, ${ }^{2}$ Department of Biology, ${ }^{3}$ Department of Mathematics and Statistics, ${ }^{4}$ Center for Interdisciplinary Biomedical Education and Research, University of Central Oklahoma, Edmond, OK, USA

Correspondence: Morshed Khandaker Department of Engineering \& Physics, University of Central Oklahoma, Box 87

Edmond, OK 73034, USA

$\mathrm{Tel}+$ I 4059745935

Fax +I 4059743812

Email mkhandaker@uco.edu
Abstract: Implant failure due to poor integration of the implant with the surrounding biomaterial is a common problem in various orthopedic and orthodontic surgeries. Implant fixation mostly depends upon the implant surface topography. Micron to nanosize circular-shaped groove architecture with adequate surface roughness can enhance the mechanical interlock and osseointegration of an implant with the host tissue and solve its poor fixation problem. Such groove architecture can be created on a titanium ( $\mathrm{Ti}$ ) alloy implant by laser peening treatment. Laser peening produces deep, residual compressive stresses in the surfaces of metal parts, delivering increased fatigue life and damage tolerance. The scientific novelty of this study is the controlled deposition of circular-shaped rough spot groove using laser peening technique and understanding the effect of the treatment techniques for improving the implant surface properties. The hypothesis of this study was that implant surface grooves created by controlled laser peen treatment can improve the mechanical and biological responses of the implant with the adjoining biomaterial. The objective of this study was to measure how the controlled laserpeened groove architecture on Ti influences its osteoblast cell functions and bonding strength with bone cement. This study determined the surface roughness and morphology of the peentreated Ti. In addition, this study compared the osteoblast cell functions (adhesion, proliferation, and differentiation) between control and peen-treated Ti samples. Finally, this study measured the fracture strength between each kind of Ti samples and bone cement under static loading. This study found that laser peen treatment on Ti significantly changed the surface architecture of the Ti, which led to enhanced osteoblast cell adhesion and differentiation on Ti implants and fracture strength of Ti-bone cement interfaces compared with values of untreated Ti samples. Therefore, the laser peen treatment method has the potential to improve the biomechanical functions of Ti implants.

Keywords: titanium, cement, interface, PMMA, fracture strength, orthopedics, laser peen, orthodontics

\section{Introduction}

The use of fixed, cemented implants was an innovation that provided more long-term stability than uncemented implants; however, clinical loosening of the cemented replacements has been reported. In USA, $\sim 600,000$ cases of poor union and 100,000 cases of nonunion of implants with the surrounding tissue are reported every year. ${ }^{1}$ Many manufacturers have recalled their hip implants (including Johnson \& Johnson [New Brunswick, NJ, USA], DePuy [West Chester, PA, USA], and Zimmer Durom [Warsaw, IN, USA]). ${ }^{2}$ A patient's age, sex, weight, diagnosis, activity level, surgery condition, and implant choice influence the longevity of the device. The primary cause of failure of cemented joint replacements is aseptic loosening of the components, 
which may arise from mechanical failure of the cement mantle surrounding the implant. ${ }^{3}$ It has been pointed out that the debonding of stem-cement interface enables gapping and sliding between the stem and the cement., ${ }^{4,5}$

A wide variety of surface modification techniques have developed with the aim of solving the implant fixation problem. Many papers have been reported the nanoscience techniques, such as plasma sintering, plasma nitridation, for obtaining a rough surface on an orthopedic or orthodontic implant. ${ }^{6,7}$ The augmentation of the surface roughness or surface energy allows for developing mechanical interlock of the implant and bone cement interface, which can improve the bonding characteristics of the implant-cement interface. ${ }^{8}$ Hosein et al ${ }^{9}$ pointed out that circumferential-grooved stems offered improved stability under compression relative to the smooth stems. It has been revealed that porous coating of the femoral stems dramatically improved push-out strengths and fatigue properties of the stem-cement interface through increasing surface roughness. ${ }^{10}$ In addition, various implant coatings, including titanium dioxide with integrated copper ions, plasma polymerized allylamine, calcium phosphate, and titanium nitride have been investigated regarding the adhesion strength and wear resistance. ${ }^{11}$ Shot peening is one of the most frequently used treatments for obtaining a rough surface of an orthopedic or orthodontic implant. ${ }^{12}$ Shot peening is used to bombard implant surfaces with chemically stable materials that will not negatively affect the biological behavior of the implant. ${ }^{13}$

Laser peening, on the other hand, is an engineered process that uses beneficial residual stresses by spot grooving to provide strong, reliable products. Laser peening has been shown to increase service lifetimes of components by more than ten times that of the as-manufactured condition, and that of parts treated with other conventional surface enhancement methods. ${ }^{13,14}$ Shot or laser peening induces a residual stress layer in the treated material because of the local plastic deformation of the metal. ${ }^{14}$ Although the variables of shot blasting are not exhaustively controlled, as opposed to laser peening, the stressed superficial layer is in compression and, consequently, an increase in the fatigue resistance of the peen-treated implant is expected. In the case of laser peening, the rapid rise of pressure generated at the surface of the workpiece by the high energy laser pulse creates a shockwave in the part. The pressure pulse typically has a length of 2-2.5 times that of the laser pulse. ${ }^{15}$ As the shockwave travels into the object, some of the energy of the wave is absorbed by the plastic deformation and beneficial residual stress accumulates in the object area. The indented surface area, dislocations, and nanocrystallization, which are typically instigated onto the implant surface by laser peening, are chemically stable materials that should not stimulate a negative response of the biological behavior of the implant. Rather the implant surface grooves created by laser peening may provide favorable depth for cell adhesion and enhance mechanical interlock between the implant and the cement, which will improve the union between the implant and the cement. The influence of the laser-peened treatment on the cell adhesion and fracture strength of Ti-cement was studied in this research.

\section{Materials and methods Materials}

This study used titanium alloy, 6Al-4V ELI as the titanium implant. Among the various Ti alloys, 6Al-4V ELI was used in this study because of its better physical and mechanical properties compared with pure $\mathrm{Ti}^{16}$ The $6 \mathrm{Al}-4 \mathrm{~V}$ ELI titanium alloy round rod has been referred in this study as the Ti rod. Two sets of round 6Al-4V ELI Ti rods (ASTM B 348 standard, grade 23, ultimate tensile strength $125 \mathrm{ksi}$, yield strength $115 \mathrm{ksi}$ ) having same diameter $(9.565 \mathrm{~mm})$, but different lengths ( 8 and $76 \mathrm{~mm}$ ) were purchased from Titanium Metal Supply, Inc., Poway, CA, USA. In this study, $9.565 \mathrm{~mm}$ diameter and $8 \mathrm{~mm}$ height Ti rod was referred to as sample set 1, whereas $9.565 \mathrm{~mm}$ diameter and $76 \mathrm{~mm}$ height peen-treated Ti rod was referred to as sample set 2 . The above two sizes of Ti rods without laser peening was referred to as control. Sample set 1 Ti rods were used for cell proliferation and differentiation tests on the Ti. Sample set 2 Ti rods were used for mechanical tests on the Ti-poly(methyl methacrylate) (PMMA) bone cement samples.

Among the many potential bone cement materials, PMMA bone cement has been successfully used in orthopedic surgeries, mostly because of its strong mechanical bonding with implant. PMMA bone cements are provided as two-component materials, a powder (PMMA beads) and a liquid (MMA monomer). These two components are mixed in a 2:1 ratio and polymerization occurs. Cobalt ${ }^{\mathrm{TM}} \mathrm{HV}$ bone cement (Biomet Inc., Warsaw, IN, USA) was used as the bone cement.

\section{Polishing protocols}

Both control and laser-peened Ti samples were polished to have uniform surface morphology for both cell adhesion and mechanical tests. For sample set 1 Ti rod samples (Figure 1A), Buehler MetaServ ${ }^{\circledR} 250$ Grinder-Polisher (Buehler, Lake Bluff, IL, USA) were used for the flat-end face polishing. The polishing process was recommended by Buehler. There were three steps for polishing all the Ti rods. The first step was to 


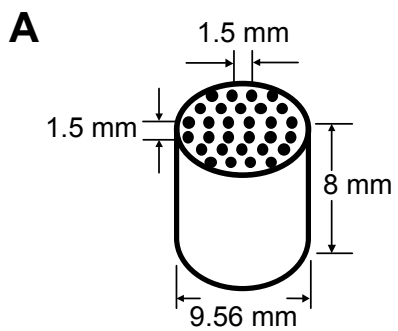

B

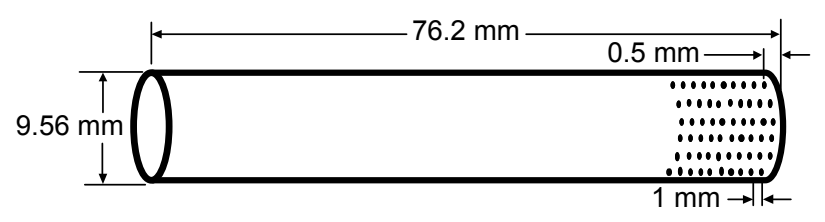

Figure I Schematic of laser peening treatment sites.

Note: (A) sample set I that was used for cell adhesion tests, and (B) sample set 2 that was used for mechanical tests.

polish using Buehler CarbiMet 2 Abrasive Discs (Buehler) for 5 minutes. During the first polishing step, a $9 \mu \mathrm{m}$ MetaDi Supreme Diamond Suspension (Buehler) was used to spray on the contact surface of the Ti rod and the polishing disc. The second polishing step used an Ultra Pad with $0.05 \mu \mathrm{m}$ MetaDi Supreme Diamond Suspension (Buehler) for an additional 5 minutes as well. The last step was using MicroCloth and MasterPrep Alumina (Buehler) for 5 minutes as well. After each step, Ti rods were cleaned by ethanol.

For sample set 2 Ti rod samples (Figure 1B), the previous three polishing steps were followed for the circumferential polishing on the Ti rods. A custom-made polishing system (Figure 2A) based on IsoMet Low Speed saw (Buehler) was used for $12 \mathrm{~mm}$ circumferential polishing from an edge. The blades of IsoMet Low Speed saw were replaced by a custom-made round disc (Figure 2B). The polishing pads (CarbiMet 2, Ultra Pad, and MicroCloth) were secured around the circumference of the round disc. Sample set $2 \mathrm{Ti}$ rod samples were fixed with the shaft of a direct current (DC) motor in a custom-made holder. The rotations of polishing disc and Ti rod were controlled by the IsoMet cutter speed and DC motor voltage, respectively. The rotational friction between Ti rod and round disc was used to polish uniformly the circumference of the Ti rod. For all three sets polishing, Buehler IsoMet cutter speed was set to $600 \mathrm{rpm}$ and DC motor voltage was set to $6.8 \mathrm{~V}$.
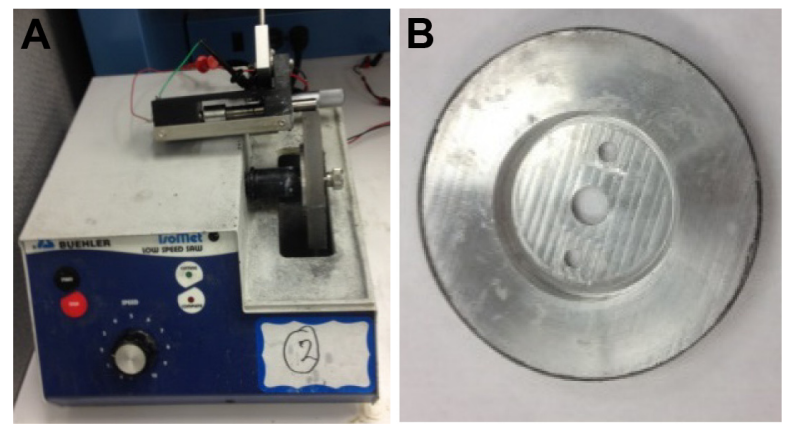

Figure 2 (A) Custom-made polishing setup using Buehler IsoMet Low Speed saw. (B) Disc used for polishing.

\section{Laser peening treatment on $\mathrm{Ti}$}

Laser peening was conducted on sample set 1 and 2 at the locations as schematically shown in Figure $1 \mathrm{~A}$ and B using Procudo ${ }^{\mathrm{TM}}$ laser peening systems (LSP Technologies, Inc., Dublin, OH, USA). LSP Technologies, Inc. is an AS9100 certified provider of laser peening. Sample 1 Ti rods were polished on the flat-end face, where sample set 2 Ti rods were polished circumferentially up to $10 \mathrm{~mm}$ from an edge. Sample set 1 had treatment applied at grid intersections ( $\sim 27$ intersections per face). Sample set 2 had ten separate bands of treatment applied to each sample at points $30^{\circ}$ around the circumference (12 points per band). A custom machine control program was developed for the laser peening procedures. All Ti rods (control and peen-treated) were sterilized by autoclaving for further experiments.

\section{Experiments}

\section{Surface roughness and morphology}

Surface morphology is an important parameter that plays a significant role in implant-cement and implant-bone adhesion. So, the influence of laser peen treatment to surface morphology was evaluated by a Leica DCM8 (Leica Microsystems, Wetzlar, Germany) surface metrology instrument using $20 \times$ bright field confocal control condition. A line scan was conducted on both samples. Roughness and depth profile along the scanned line were compared.

\section{Osteoblast culture and preparation}

Rat osteoblast cells (R-OST-583; Lonza, Basel, Switzerland) were cultured at $\log$ phase growth in standard culture conditions $\left(37^{\circ} \mathrm{C}\right.$ in a $5 \% \mathrm{CO}_{2}$ incubator on tissue culture dishes) using Dulbecco's Modified Eagle's Medium/high glucose $+5 \%$ fetal bovine serum and 1\% Antibiotic Antimycotic (SigmaAldrich Co., St Louis, MO, USA). Cells were dissociated using $1 \times$ trypsin/ethylenediaminetetraacetic acid solution (SigmaAldrich Co.) for 5 minutes at room temperature, followed by serum inactivation. Cells were counted using a hemocytometer, collected by centrifugation, and resuspended at a concentration of 35,000 cells per $400 \mu \mathrm{L}$ of growth media. 


\section{Cell adhesion and proliferation tests}

Sample set 1 was used to test the effect laser peen-treated Ti on osteoblast adhesion and proliferation. Custom-designed silicone molds (Figure 3) were used to hold the Ti samples (control and laser peen-treated) and work as a cell culture well plate. Thirty-five thousand osteoblast cells were seeded on each Ti sample and returned to culture. Before adding the cells, $200 \mu \mathrm{L}$ of culture media was added onto each Ti samples in the silicone mold to minimize cell attachment to the edge. After 24 hours of culture, cells were cultured for an additional 24 hours with a modified nucleotide (ethynyl deoxyuridine, $20 \mu \mathrm{M}$ ) to determine proliferation, as previously described ${ }^{17}$ using a purchased kit (Click-iT; Thermo Fisher, Carlsbad, CA, USA). Samples were fixed for 20 minutes with $4 \%$ paraformaldehyde, and stained as per the Click-iT kit instructions. Nuclei and proliferated cells were identified using images captured with an Olympus IX-71 inverted fluorescent microscope ( $\times 10$ magnification), Olympus DP72 camera, and CelSens software (Olympus Corporation, Shinjuku, Tokyo, Japan). The quantitative measurements of cell adhesion and proliferation on the control and treated Ti surfaces were determined from the captured images. The number of cells adhered and the number of cells proliferated after adhesion to each samples were determined from the captured images using the ImageJ software program (http://imagej.nih.gov/ij/). The adhered cell density was calculated by dividing the

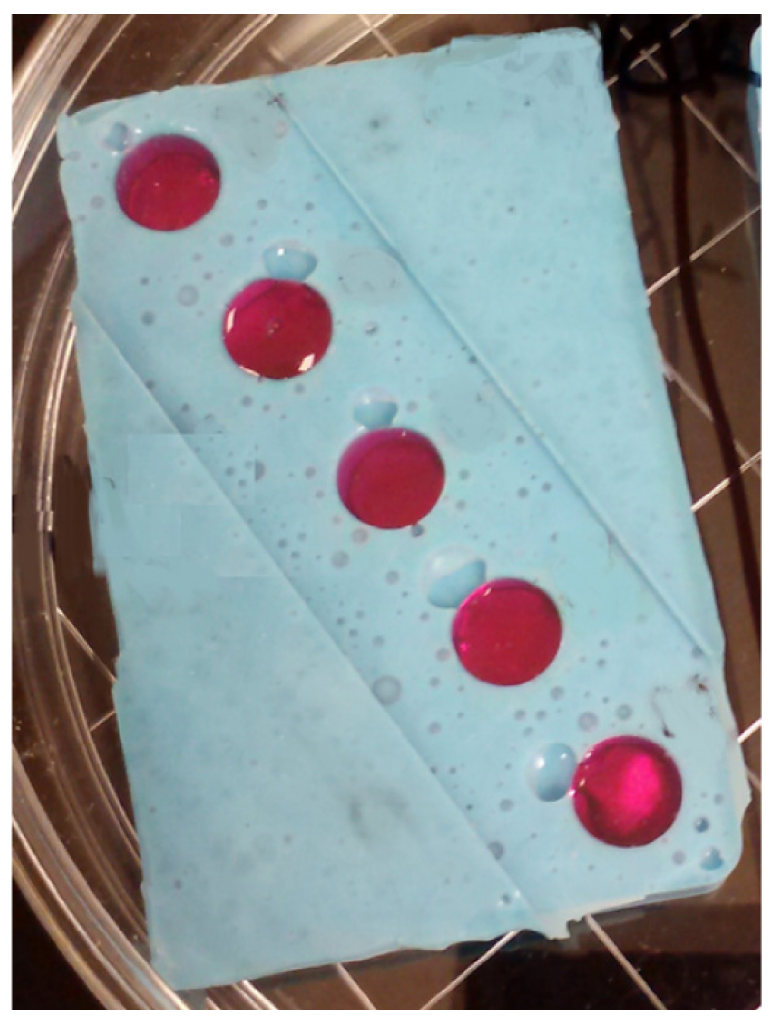

Figure 3 Titanium with cells in silicone molds. number of cells adhered per image by the calculated area of the image (calculated to be $1.473 \mathrm{~mm}^{2}$ by ImageJ). The percentage of proliferation was calculated by the following equation: (the number of cells proliferated after adhesion/the number of cells adhered to surface) $\times 100 \%$.

\section{Cell differentiation test}

Parallel samples similar to those tested for proliferation were cultured for 7 days and prepared for immunostaining to determine hydroxyapatite mineralization. Half of the culture media was changed and replaced with fresh media after 3 days. Cells were fixed with $4 \%$ paraformaldehyde for 20 minutes. To stain these samples, OsteoImage ${ }^{\mathrm{TM}}$ Mineralization Assay kit from Lonza was used according to vendor protocol. Stained samples were immersed in $80 \%$ glycerol in phosphate buffered saline containing $1 \mu \mathrm{M}$ solution of DAPI (4', 6-diamidino2-phenylindole). Samples were viewed with an IX-71 epifluorescent inverted microscope. Images were captured with an Olympus DP72 camera and CelSens software. The qualitative measurements of cell differentiation on the control and treated Ti surfaces were determined from the captured images.

\section{Mechanical tests on $\mathrm{Ti} /$ cement samples}

Sample set 2 control and laser peen-treated Ti rods were used to find the effect of laser peen treatments on the bonding strength between the Ti rods and cement under static loading. A Test Resources (Shakopee, MN, USA) universal testing machine (UTM) was used for the mechanical tests. The top gripper of the UTM held the Ti rods (Figure 4). A 3D printed (Dimension elite 3D printer; Stratasys, Edina, MN, USA) cylindricalshaped cement holding cup was fabricated to encapsulate the

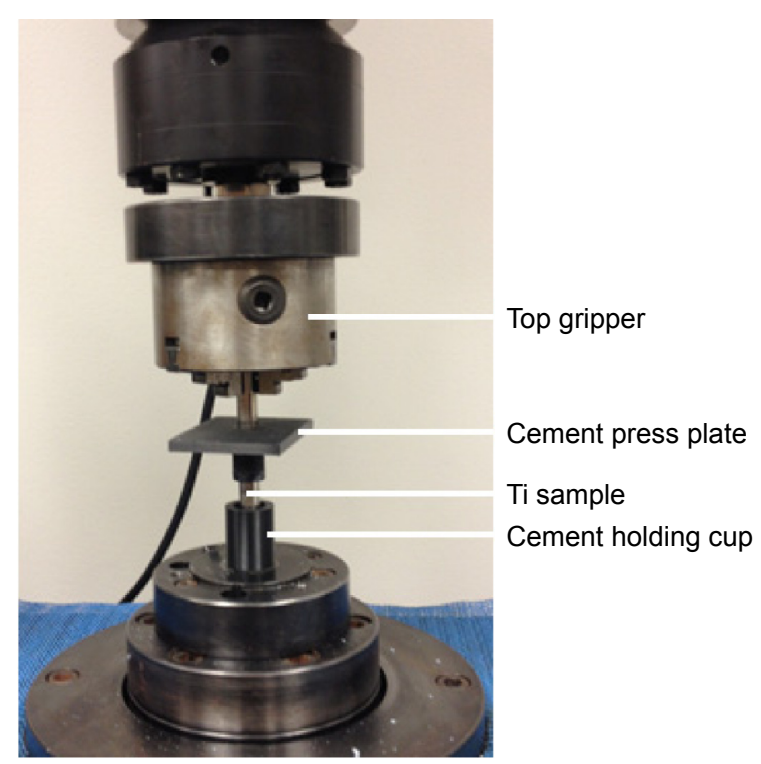

Figure 4 Static tests setup on universal testing machine. 


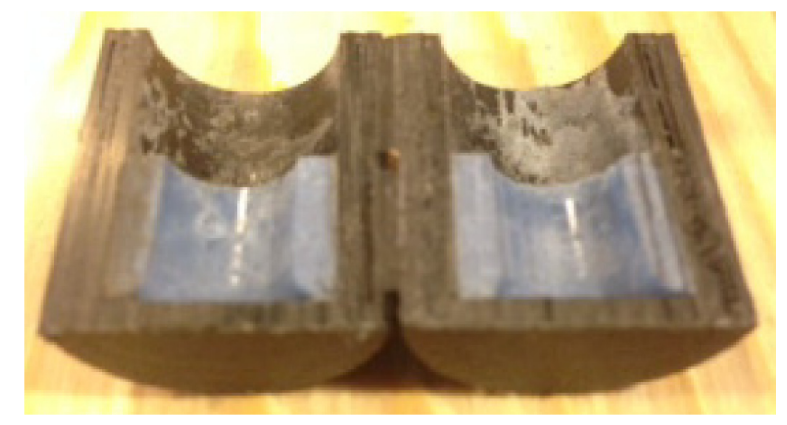

Figure 5 Measurement of the cement height by cutting cement holding cup into half using the band saw machine.

Ti rod with $\sim 2.8 \mathrm{~mm}$ thick and $12 \mathrm{~mm}$ height PMMA cement layer. The cup was placed at the center of the bottom base of the UTM with the help of another 3D printed plate, referred in the Figure 4 as cement press plate. The plate has a tube extruded from the center of the plate. The dimension of the inside and outside diameter of the tube was such that the Ti rod and cup fit within the tube. The plate slid onto the Ti rod. The Ti rod with the plate was jogged down until the Ti rod touched the bottom surface of the cup. According to Cobalt $\mathrm{HV}$ bone cement manufacturer package recommendations, PMMA cement should be prepared by hand mixing $2.2 \mathrm{~g}$ of PMMA powder with $1.1 \mathrm{~mL}$ of MMA monomer using powder:monomer ratio of 2:1. PMMA cement paste was poured into the gap between the Ti and cup. The plate was pressed by hand to encapsulate the bottom end of the Ti rod

\section{A}

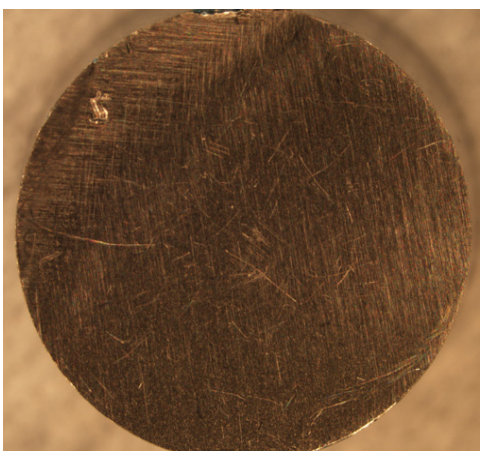

\section{C}

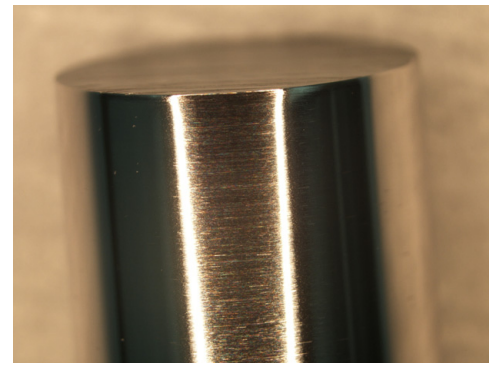

with cement. The plate was bolted with the bottom gripper of the UTM. After curing the cement for 15 minutes, static tests were conducted at strain rate $0.05 \mathrm{~mm} / \mathrm{s}$ on the sample to find fracture strength of the Ti/PMMA samples. Load and displacement data during the test were recorded. The maximum pullout force, $F$, was determined from the recorded data. The cup was cut in half to measure the height of the cement layer of the Ti/PMMA samples (Figure 5). The average cement layer height, $L$, was measured. Fracture strength of the Ti/PMMA interface was calculated by dividing the maximum pull-out force by the surface area of the implant in contact with the cement $(\pi D L)$, where $D$ is the diameter of the Ti rod.

\section{Statistical analysis}

A one-factor analysis of variance with subsampling assuming unequal variances was performed in SAS v. 9.3 (SAS Institute Inc., Cary, NC, USA) using proc mixed to determine if there is a significant difference in the mean cell densities of the control and peen-treated groups. An independent samples $t$-test, assuming unequal variances, was performed using SAS v. 9.3 to determine if there is a significant difference in the mean fracture strength of the control and peen-treated groups.

\section{Results}

Figure 6A-D shows the stereomicroscope images of a control and laser peen-treated sample sets 1 and 2 used for the osteoblast cell function and mechanical tests, respectively.

\section{B}

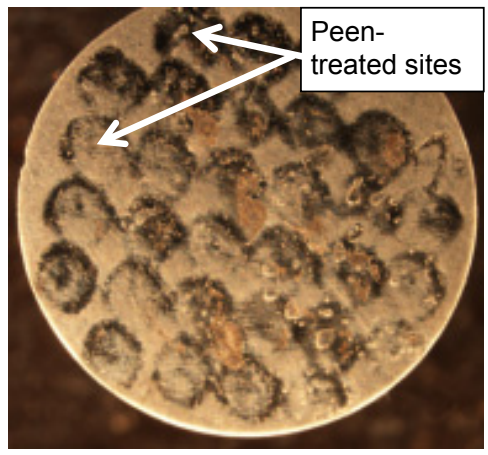

D

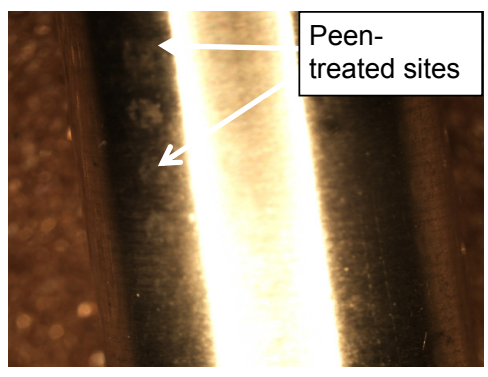

Figure 6 Laser-peened Ti samples.

Notes: Sample set I used for osteoblast cell function studies: (A) control and (B) peen-treated sample set. Sample set 2 used for static tests: (C) control and (D) peentreated samples.

Abbreviation: Ti, titanium. 
A clear difference of surface topography between the control and laser peen-treated samples can be seen from the images. Laser-peened indentation sites were clearly visible for both kinds of samples. Confocal laser microscopic image of a peen-treated sample used for cell studies shows a clear topographical difference between non-peen-treated and peentreated sites (Figure 7A). The depth ( $z$-axis height) line profile along a vertical path that covered both non-peen-treated and peen-treated sites (Figure 7B) showed the quantitative difference of surface indentation depth between the sites. Figure 7B shows a difference of $1.49 \mu \mathrm{m}$ in the average depth values between non-peen-treated and peen-treated sites for same linear distance $(190.30 \mu \mathrm{m})$. In addition, the surface roughness of laser-peened samples was found to be higher compared to control samples (Table 1).

Cells adhesion and proliferation successfully occurs on the surface of all types of Ti samples as shown in Figure 8. Both samples demonstrated nonproliferating cells (blue nuclei) and proliferating cells (green nuclei). There is significant difference in the mean cell densities (combined blue and green nuclei) of the control and peen-treated Ti sample groups $(F(1,34)=5.81, P=0.021)$ as shown in Figure 9. These results suggested that laser peen treatment has a positive influence on the osseointegration with the Ti surface. Because osteoblasts are anchorage-dependent, increased roughness of surface area would allow the osteoblasts to adhere more often and tighter, allowing a higher rate of attachment, and perhaps persistence, to occur.

Figure 10 shows that cells successfully differentiate between both control and a peen-treated Ti samples. Untreated Ti samples demonstrated a weak pericellular stain near many osteoblast nuclei (Figure 10A). Peen-treated Ti samples demonstrated a brighter pericellular staining pattern near most osteoblast nuclei (Figure 10B) as previously reported. ${ }^{18}$

The comparison of the load-displacement plots of a control and peen-treated specimen is shown in Figure 11. A sudden increment of load was observed for all specimens at the initial stage. Such high initial load was higher for peentreated samples compared to the control samples. After the high initial load, the slope of the load versus displacement value for all control samples was higher at the initial stage than the slope at failure stage (1.66 vs $1.36 \mathrm{~N} / \mathrm{mm}, 1.13 \mathrm{vs}$ $1.06 \mathrm{~N} / \mathrm{mm}$, and $1.69 \mathrm{vs} 1.04 \mathrm{~N} / \mathrm{mm}$ ). In contrast, after the high initial load, the slope of the load versus displacement value for all laser peen-treated samples was lower at the initial stage than the slope at failure stage ( $1.2 \mathrm{vs} 2.1 \mathrm{~N} / \mathrm{mm}, 2.64 \mathrm{vs}$ $3.12 \mathrm{~N} / \mathrm{mm}$, and $1.11 \mathrm{vs} 1.24 \mathrm{~N} / \mathrm{mm}$ ). This result occurs due to the surface topographical variation between the control and laser peen-treated samples. The increment of load with displacement behaved linear until the onset of cracking, which is
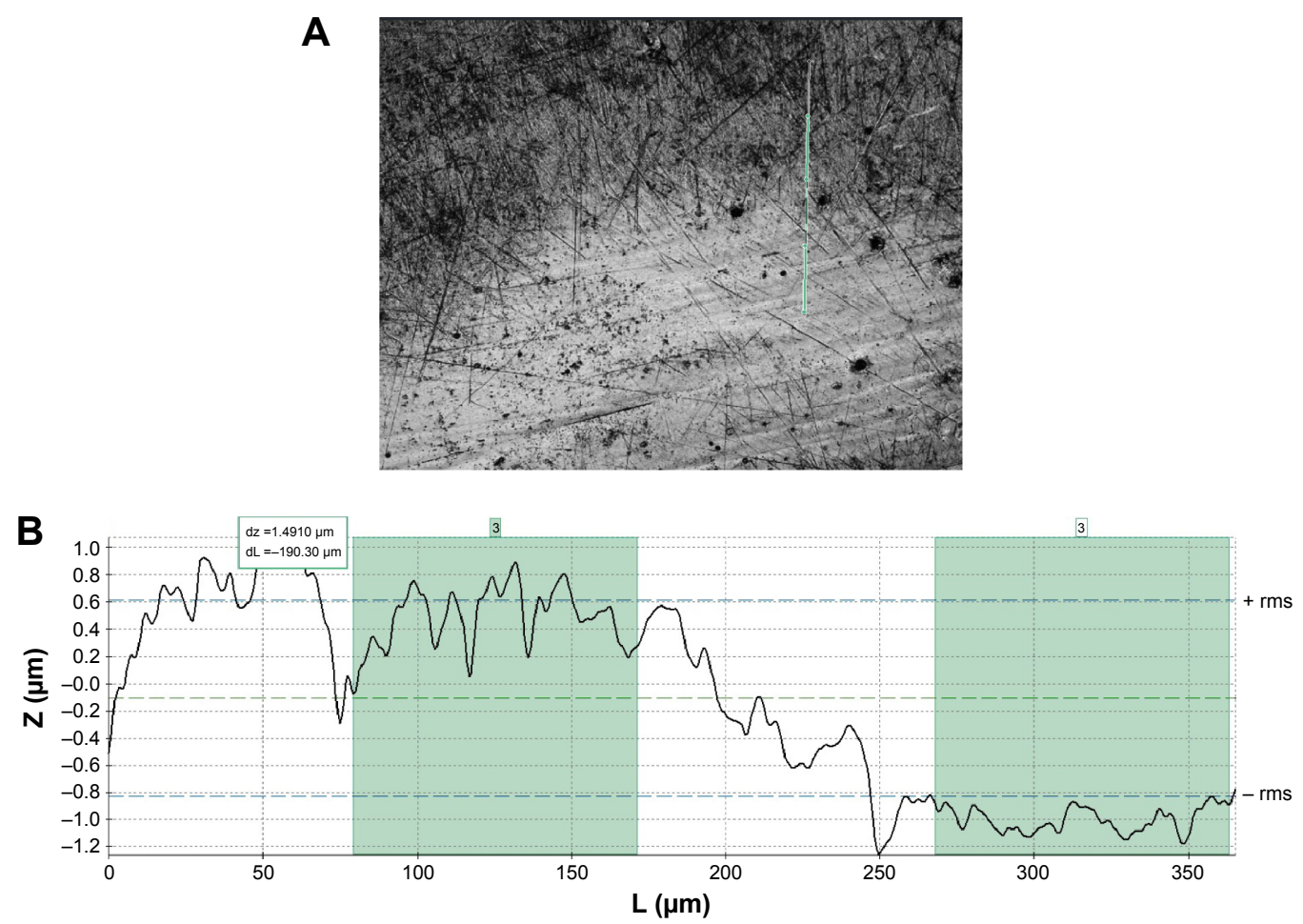

Figure 7 (A) Confocal area scan $(876.55 \mu \mathrm{m} \times 659.83 \mu \mathrm{m})$ image of laser peen-treated sample. (B) Confocal Z scan (I30 $\mu \mathrm{m})$ to find the depth profile along a vertical line indicated in (A). 
Table I Difference of roughness between control and laser peen-treated samples

\begin{tabular}{lll}
\hline Descriptions & Control $(\mu \mathrm{m})$ & Peen-treated $(\mu \mathrm{m})$ \\
\hline Profile length & 178.09 & 365.13 \\
$R_{a}$ & 0.10 & 0.66 \\
$R_{q}$ & 0.13 & 0.72 \\
$R_{p}$ & 0.28 & 1.18 \\
$R_{v}$ & 0.35 & 1.15 \\
$R_{z}$ & 0.63 & 2.33 \\
\hline
\end{tabular}

observed in the figure by the nonlinearity (fracture initiation) point of the load-displacement curve. Also, Figure 11 shows that the peen-treated specimen required more fracture load than the control specimen. Results show that the amount of displacement before the initiation of crack at the interface is different between control and peen-treated samples. The values of fracture strength of both samples were calculated. The specimen static tests, experimental parameters, and mean fracture strengths for the control and peen-treated groups are displayed in Figure 12 and Table 2. There was a significant difference in the mean fracture strengths of the
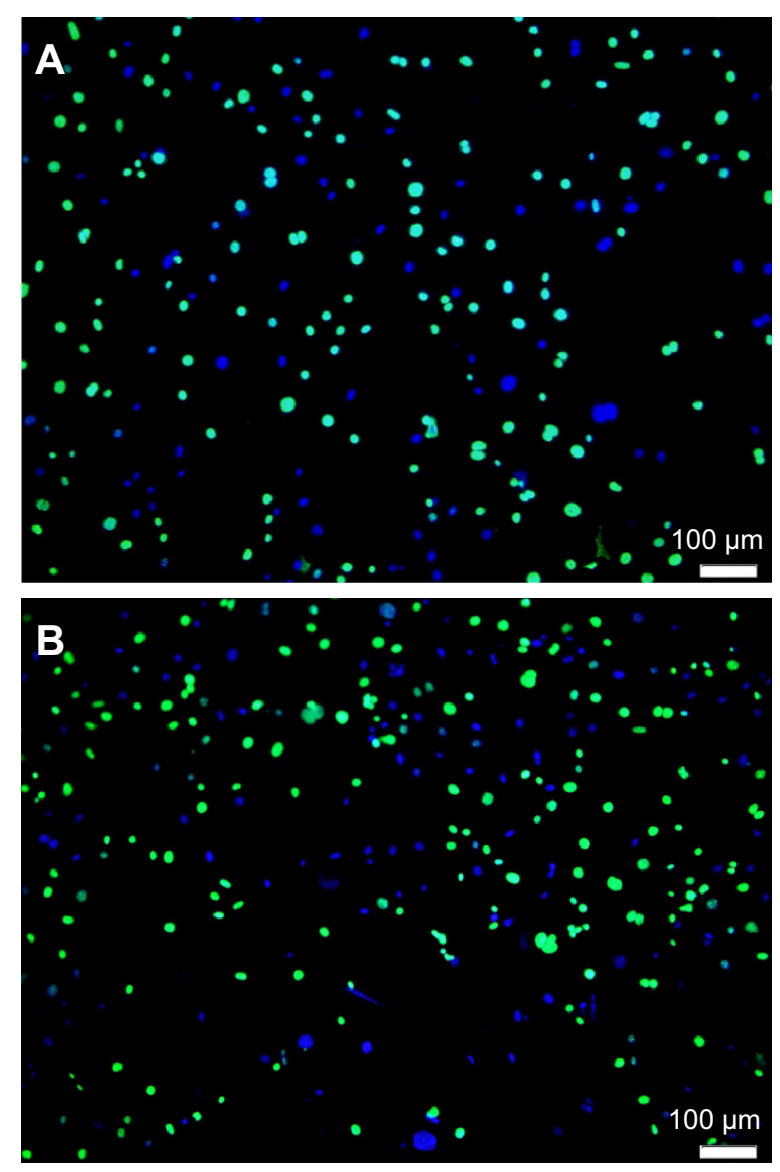

Figure 8 Fluorescent microscope image after cell adhesion and proliferation experiments on a $(\mathbf{A})$ control and $(\mathbf{B})$ peen-treated Ti sample. Abbreviation: $\mathrm{Ti}$, titanium.

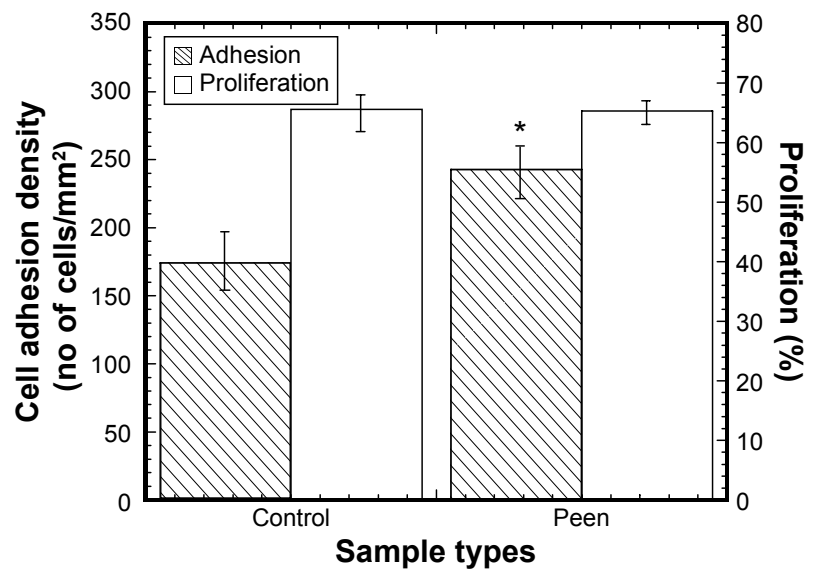

Figure 9 Mean cell adhesion density and percentage of proliferation after attachment for the control and laser peen-treated samples.

Note: $* P<0.05$ (compared to control).

groups ( $t=4.24, P=0.036)$. Since higher fracture strength of a bimaterial interface means better union of the interface, therefore, the previous results suggested that peen treatment has a positive influence on the union between $\mathrm{Ti}$ and cement interfaces.
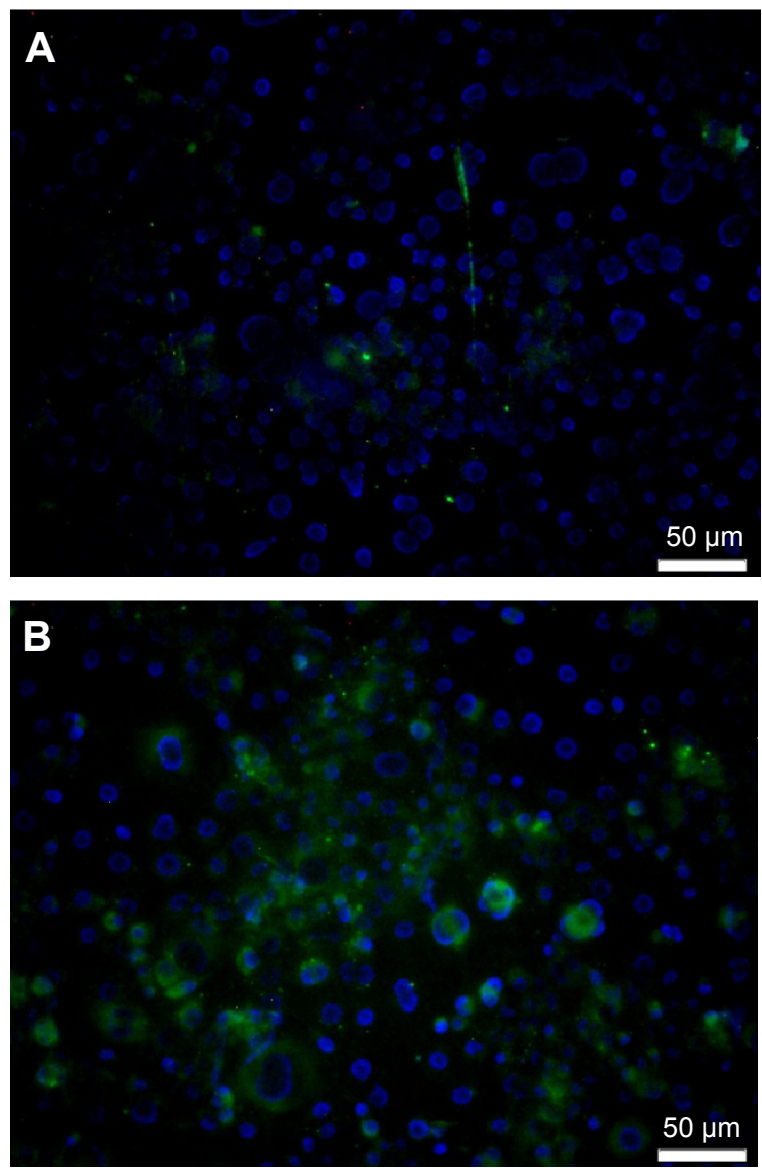

Figure 10 Fluorescent microscope image after cell differentiation experiments on a (A) control and (B) peen-treated Ti sample.

Abbreviation: $\mathrm{Ti}$, titanium. 


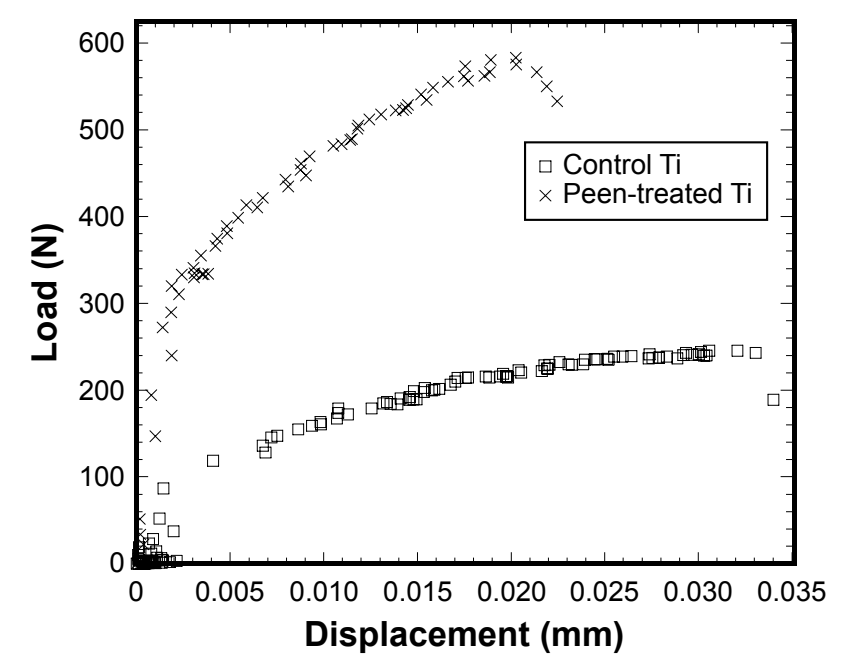

Figure II Load-displacement curves of three samples during the static tests on Ti-cement interfaces.

Abbreviation: $\mathrm{Ti}$, titanium.

\section{Discussion}

Laser peening treatment on a Ti alloy demonstrated better cell adhesion and bonding strength between the metal and cement compared to control. Higher surface roughness, surface energy, pores, dislocation arrangement, and nanocrystallization are some of the potential causes for the biological and mechanical improvements.

Surface roughness measurements showed that the roughness achieved by polishing and subsequent laser peening is much higher than surface roughness achievable by simple polishing (Table 1). Since there is a positive relation between surface roughness and cell response, ${ }^{19}$ the polishing steps used in this study might be favorable as a surface treatment before using laser peening. Greater osteoblast cell adhesion

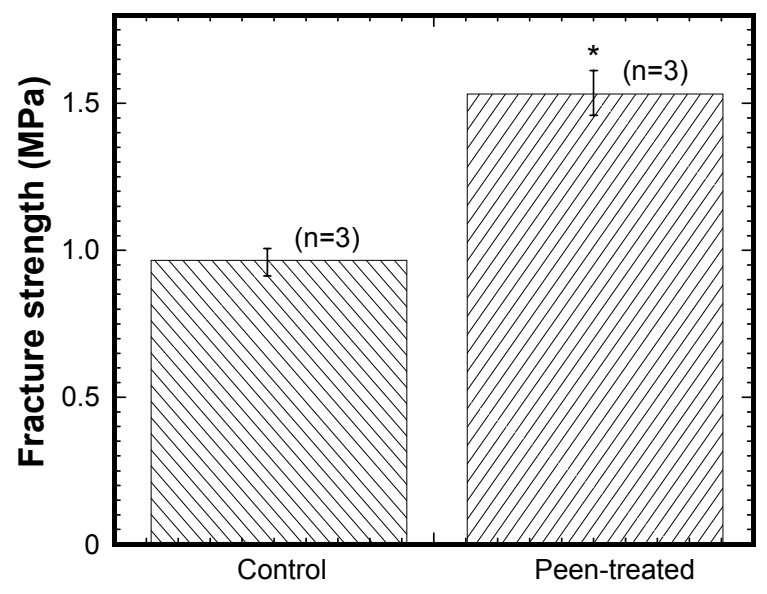

Figure 12 Bar diagram of the variation of fracture strength of control samples (Ti) with peen-treated samples.

Notes: Data presented as mean \pm standard error of mean; $\mathrm{n}=3$ for control and laser peen-treated samples. $* P<0.05$ (compared to control).

Abbreviation: $\mathrm{Ti}$, titanium.
Table 2 Summary statistics for static tests experimental data by group

\begin{tabular}{lll}
\hline Parameters descriptions & Control & Peen-treated \\
\hline No of samples tested & 3 & 3 \\
Height of implant-cement interface & $10.23 \pm 0.18$ & $11.48 \pm 0.46$ \\
Contact area & $307.34 \pm 5.47$ & $338.76 \pm 13.76$ \\
Fracture load & $329.21 \pm 41.05$ & $527.01 \pm 39.77$ \\
Fracture strength & $1.05 \pm 0.14$ & $1.54 \pm 0.18$ \\
\hline
\end{tabular}

with greater nanosurface features (nanosurface roughness and surface energy) could be entirely due incrementally increased surface area by the laser peening process. Cells in both control and peen-treated conditions were able to bind and persist. A recent work by Shen et $\mathrm{al}^{20}$ has shown that osteoblasts attach and elongate atop titanium surfaces. In this study, similar cell behavior is present, suggesting that osteoblasts are able to attach, elongate, and migrate. More cells were adhered on the laser peen-treated surfaces than control. The enhanced surface roughness and texture due to laser peen treatment may be potential reason for this phenomena.

Osteoblasts, like other mesenchymal-derived cells, require anchorage in order to survive, proliferate, and differentiate. Here we showed that osteoblasts survive and proliferate under both test conditions, Ti-only and peentreated Ti substrates. The samples were assessed during the second day of culture in conditions that favor proliferation. Longer term culture would likely slow down proliferation after cell-cell contacts become saturated.

Osteoblast differentiation is correlated with deposition of mineralized matrix materials such as hydroxyapatite. ${ }^{21}$ We demonstrated the presence of mineralized deposits at the periphery of cultured osteoblasts in both test conditions after 7 days of culture. The staining was qualitatively more intense in the peen-treated samples. This suggests that the peen treatment provides the osteoblasts an appropriate microenvironment to stimulate bone deposition earlier or greater than under nontreated conditions. It is unknown whether longer term culture would allow the untreated Ti time to achieve deposition similar to the peen-treated samples. Lim et $\mathrm{al}^{22}$ study suggested that surface energy effects on the osteoblast cell differentiation, especially mineralization, may be correlated with surface energy-dependent changes in spatial cell attachment and growth. It is likely that, in addition to mineralization, osteoblasts under the tested conditions will secrete bone-related matrix proteins such as osteonectin and osteopontin. ${ }^{23}$ Future studies will determine how protein expression is affected by these treatment conditions.

The difference in fracture strength between control and laser peen-treated samples is mainly due to the difference 
in surface texture between laser peen and control samples. The surface texture of the metal will allow a mechanical interlock between the metal and cement. This phenomenon was made clear from the study conducted by Davies and Harris, ${ }^{24}$ which showed that increasing the mechanical interlock of the cement to metal by adding indentations to the surface or using a porous surface significantly increased the static and the number of fatigue push-out cycles required for the failure of the metal-cement interfaces. Fracture strength of the implant-cement interface can be defined as the maximum resistance to the propagation of an interface crack originating at the interface. The weakest spots of implant-cement interfaces are the natural flaws along the interface created during cement polymerization. Implant surface roughness and cement mechanical properties on interfaces may affect the propagation of these flaws, that is fracture toughness of the implant-cement interface. ${ }^{25,26}$ Increased surface roughness due to laser peen treatment led to a decrease of micromovement of cracks at the implant-cement interface, thus the load and elongation was increased at the fracture. Further improvement of the fracture strength of the implant-cement interface can be achieved by optimization of the laser peening pattern and depth.

Surface pores, dislocation arrangement, and nanocrystallization are triggered by laser peening. ${ }^{15}$ Bone ingrowth into pores causes interlocking of the surrounding bone tissue with the implant, and may result in improved in vivo biomechanical stability and higher resistance to fatigue loading. ${ }^{27}$ A study by Götz et $\mathrm{al}^{28}$ showed that initial bone ingrowth is preferentially directed toward the pores of the implant. Thus, an added advantage is that laser-textured implants with open pores may provide improved stability during early stages of osseointegration. Surface dislocation arrangement and nanocrystallization triggered by laser peening can effectively enhance surface mechanical properties such as elastic modulus and wear resistance. ${ }^{29}$ Our previous studies found that, the elastic modulus of cement and wear resistance of an implant due to nanofiber coating on the implant controls the bonding strength between the implant and the cement. ${ }^{25,30}$ Thus, laser peen-created surface dislocation arrangement and nanocrystallization on Ti-based alloys may affect the bonding strength between $\mathrm{Ti}$ and cement.

Laser peening can improve the mechanical strength and introduce a micropattern into the biomedical implant materials such as stainless steel, ${ }^{31} \mathrm{Ti}^{20,32}$ The microhardness, compressive residual stress, and microstructure introduced by laser peening created plastic deformation on the implant surface and has higher union capability with joining biomaterial (PMMA used in our study) than nontreated implants.
The laser peening treatment on an implant is permanent and cannot be removed during the insertion or the extraction of the implant during surgeries. The biomedical performance of the patterned surface created by laser peening has higher cell attachment than nontreated titanium implants. An enhancement of the abrasion and corrosion resistance of stainless steel implant surface is possible with the application of laser peening as found by Lim et al. ${ }^{31}$

The limitation of the study was that the area topography scan was conducted using confocal laser microscope on only one control and peen-treated samples to evaluate the topographical differences between the samples. The reason for not using the multiple samples was that laser peen-treated samples create distinct circular textures, which are not present in control samples. Also line topography scan was conducted using confocal laser microscope only one peen-treated sample to evaluate the topographical differences between the nonpeen and peen-treated sites. The reason for using one site for evaluation was that each of the laser-peened sites was created by same amount of energy laser pulse applied to each of the sites. Therefore, the authors assumed that all laser-peened sites should have the same depth characteristics.

The results of the study go toward the phenomena that the increase of the surface roughness increase the effective area available for better cohesion. The effect of nano-inclusions on materials' strength and toughness has been reported in several literatures. ${ }^{33,34}$ Charitidis et $\mathrm{al}^{33}$ and Karakasidis et $\mathrm{al}^{34}$ studies found that fracture behavior of glass-ceramic materials are controlled by the size of crystalline inclusions to the material as well as the width of the grain boundary regions, respectively. The laser peen treatment leads also to better fracture behavior, which, to a certain extent, goes in the direction of experimental and theoretical observations about crack propagation. The crack propagation is affected by the local surface area of the inclusion and the matrix and the cohesion energy of the inclusions caused by the laser treatment. In addition, the results of the study go toward the understanding of currently available nanoscience techniques for improving the implant surface properties and their effects for the development of novel materials for orthopedic applications. ${ }^{6}$

\section{Conclusion}

The objectives of the study were the evaluation of the surface morphology, osteoblast cell adhesion, and interface fracture strength of Ti due to laser peen treatment on a Ti implant. This study concluded that the laser peen treatment significantly changed the nanoarchitecture of Ti samples, which lead to the enhancement of the osteoblast cell adhesion on Ti surfaces and 
mechanical stability of Ti with PMMA bone cement. These studies concluded that laser peen treatment on the Ti alloy can potentially improve the union between titanium-cement interfaces. Since the cell adhesion of all anchorage-dependent cells is related to protein adsorption, ${ }^{35}$ future studies will demonstrate the importance of nanosurface roughness to induce greater cell adhesion through the adsorption of multiadhesive proteins like osteonectin and osteopontin. In addition, future studies will determine the effect of laser peening on the fatigue life of the titanium and bone cement interface.

\section{Acknowledgments}

This publication was made possible by Grant Number 8P20GM103447 from the NIH, on-campus RCSA faculty grant from the University of Central Oklahoma Office of Research and Grants, and the CURE-STEM program from the UCO College of Mathematics and Science.

\section{Disclosure}

The authors report no conflicts of interest in this work.

\section{References}

1. Bishop JA, Palanca AA, Bellino MJ, Lowenberg DW. Assessment of compromised fracture healing. J Am Acad Orthop Surg. 2012;20(5): 273-282.

2. idataresearch.com, U.S. Orthopedic Trauma Devices Market; 2013.

3. Jeffers JR, Browne M, Lennon AB, Prendergast PJ, Taylor M. Cement mantle fatigue failure in total hip replacement: experimental and computational testing. J Biomech. 2007;40(7):1525-1533.

4. Ramos A, Simões JA. The influence of cement mantle thickness and stem geometry on fatigue damage in two different cemented hip femoral prostheses. J Biomech. 2009;42(15):2602-2610.

5. Gravius S, Wirtz DC, Siebert $\mathrm{CH}$. In vitro interface and cement mantle analysis of different femur stem designs. J Biomech. 2008;41: 2021-2028.

6. Christensen SL, Padmos JD, Chatt A, Zhang P. Preparation and orthopaedic applications of nanoparticle-modified biocompatible surfaces. Rev Nanosci Nanotechnol. 2013;2:63-78.

7. Al Mansour F, Karpukhina N, Grasso S, Wilson RM, Reece MJ, Cattell MJ. The effect of spark plasma sintering on lithium disilicate glass-ceramics. Dent Mater. 2015;31(10):e226-e235.

8. Ohashi KL, Romero AC, McGowan PD, Maloney WJ, Dauskardt RH. Adhesion and reliability of interfaces in cemented total joint arthroplasties. J Orthop Res. 1998;16:705-714.

9. Hosein YK, Graham JW, Dunning CE. The effect of stem circumferential grooves on the stability at the implant-cement interface. $J$ Med Devices. 2014;8:014504.

10. Manley MT, Stern LS, Gurtowski J. The load carrying and fatigue properties of the stem-cement interface with smooth and porous coated femoral components. J Biomed Mater Res. 1985;19:563-575.

11. Fritsche A, Haenle M, Zietz C. Mechanical characterization of antiinfectious, anti-allergic, and bioactive coatings on orthopedic implant surfaces. J Mater Sci. 2009;44:5544-5551.

12. Javier Gil F, Planell JA, Padros A, Aparicio C. The effect of shot blasting and heat treatment on the fatigue behavior of titanium for dental implant applications. Dent Mater. 2007;23(4):486-491.

13. Laamouri A, Fathallah R, Sidhom H, Braham C. High cycle fatigue behavior prediction of shot peened parts. Int J Fatigue. 2004;26: 1053-1067.
14. Nalla RK, Atenberger I, Noster U, Liu GY, Scoltes B, Ritchie RO. On the influence of mechanical surface treatment-deep rolling and laser shock peening-on the fatigue behavior of Ti6Al4V at ambient and elevated temperatures. Mater Sci Eng A. 2003;335:216-230.

15. I. LSP Technologies. (2013). How Laser Peening Works. Available from: http://www.lsptechnologies.com/how-laser-peening-works.php

16. Hamanaka H, Doi H, Yoneyama T, Okuno O. Dental casting of titanium and Ni-Ti alloys by a new casting machine. J Dent Res. 1989;68(11): 1529-1533.

17. Vaughan MB, Odejimi TD, Morris TL, Sawalha D, Spencer CL. A new bioassay identifies proliferation ratios of fibroblasts and myofibroblasts. Cell Biol Int. 2014;38:981-986.

18. Langenbach F, Berr K, Naujoks C, et al. Generation and differentiation of microtissues from multipotent precursor cells for use in tissue engineering. Nat Protoc. 2011;6:1726-1735.

19. Havlikova J, Strasky J, Vandrovcova M, et al. Innovative surface modification of Ti-6Al-4V alloy with a positive effect on osteoblast proliferation and fatigue performance. Mater Sci Eng C Mater Biol Appl. 2014;39:371-379.

20. Shen N, Ding H, Bowers R, et al. Surface micropatterning of pure titanium for biomedical applications via high energy pulse laser peening. J Micro Nano Manuf. 2015;3:011005.

21. Hashemibeni B, Jafary F, Esmaeil, et al. Comparison of phenotypic characterization between differentiated osteoblasts from stem cells and calvaria osteoblasts in vitro. Int J Prev Med. 2013;4:180-186.

22. Lim JY, Shaughnessy MC, Zhou Z, Noh H, Vogler EA, Donahue HJ. Surface energy effects on osteoblast spatial growth and mineralization. Biomaterials. 2008;29:1776-1784.

23. Crockett JC, Rogers MJ, Coxon FP, Hocking LJ, Helfrich MH. Bone remodelling at a glance. J Cell Sci. 2011;124:991-998.

24. Davies JP, Harris WH. Strength of cement-metal interfaces in fatigue: comparison of smooth, porous and precoated specimens. Clin Mater. 1993;12:121-126.

25. Khandaker M, Utsaha KC, Morris T. Interfacial fracture toughness of titanium-cement interfaces: effects of fibers and loading angles. Int $J$ Nanomedicine. 2014;9:1689-1697.

26. Walsh WR, Svehla MJ, Russell J, et al. Cemented fixation with PMMA or Bis-GMA resin hydroxyapatite cement: effect of implant surface roughness. Biomaterials. 2004;25:4929-4934.

27. Jinno T, Goldberg VM, Davy D, Stevenson S. Osseointegration of surface-blasted implants made of titanium alloy and cobalt-chromium alloy in a rabbit intramedullary model. J Biomed Mater Res. 1998;42: 20-29.

28. Götz HE, Müller M, Emmel A, Holzwarth U, Erben RG, Stangl R. Effect of surface finish on the osseointegration of laser-treated titanium alloy implants. Biomaterials. 2004;25:4057-4064.

29. Wu S, Liu X, Yeung KWK, et al. Surface nano-architectures and their effects on the mechanical properties and corrosion behavior of Ti-based orthopedic implants. Surf Coat Technol. 2013;233:13-26.

30. Khandaker M, Li Y, Morris T. MgO micro/nano particles for the improvement of cement-bone interface. J Biomech. 2013;46:1035-1039.

31. Lim H, Kim P, Jeong H, Jeong S. Enhancement of abrasion and corrosion resistance of duplex stainless steel by laser shock peening. J Mater Processing Technol. 2012;121:1347-1354.

32. Qiao H, Zhao J, Gao Y. Experimental investigation of laser peening on TiAl alloy microstructure and properties. Chin J Aeronaut. 2015;28:609-616.

33. Charitidis CA, Karakasidis TE, Kavouras P, Karakostas T. The size effect of crystalline inclusions on the fracture modes in glass-ceramic materials. J Phys Condens Matter. 2007;19:266209.

34. Karakasidis TE, Charitidis CA. Influence of nano-inclusions' grain boundaries on crack propagation modes in materials. Mater Sci Eng B. 2011;176:490-493.

35. Khang D, Kim SY, Liu-Snyder P, Palmore GTR, Durbin SM, Webster TJ. Enhanced fibronectin adsorption on carbon nanotube/ poly(carbonate) urethane: independent role of surface nano-roughness and associated surface energy. Biomaterials. 2007;28:4756-4768. 
International Journal of Nanomedicine

Dovepress

\section{Publish your work in this journal}

The International Journal of Nanomedicine is an international, peerreviewed journal focusing on the application of nanotechnology in diagnostics, therapeutics, and drug delivery systems throughout the biomedical field. This journal is indexed on PubMed Central, MedLine, CAS, SciSearch $®$, Current Contents $® /$ Clinical Medicine,
Journal Citation Reports/Science Edition, EMBase, Scopus and the Elsevier Bibliographic databases. The manuscript management system is completely online and includes a very quick and fair peer-review system, which is all easy to use. Visit http://www.dovepress.com/ testimonials.php to read real quotes from published authors.

Submit your manuscript here: http://www.dovepress.com/international-journal-of-nanomedicine-journal 